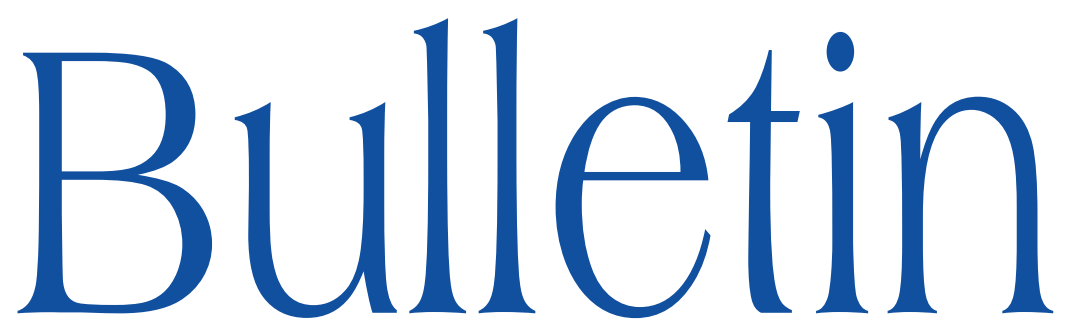

de la SOCIÉTÉ MATHÉMATIQUE DE FRANCE

\title{
SUR LES FONCTIONS À LIEU SINGULIER DE DIMENSION 1
}

\author{
Daniel Barlet
}

\section{Tome 137}

Fascicule 4

2000 


\title{
SUR LES FONCTIONS À LIEU SINGULIER DE DIMENSION 1
}

\author{
PAR DANIEL BARLET
}

En hommage à Bernard Malgrange

RÉSumÉ. - Dans notre article [6] nous avons construit, pour une classe assez large de germes de fonctions holomorphes $f:\left(\mathbb{C}^{n+1}, 0\right) \rightarrow(\mathbb{C}, 0)$ à lieu singulier $S:=\{d f=0\}$ de dimension 1 des invariants analytiques qui généralisent le réseau de Brieskorn d'un germe à singularité isolée. Dans cet article nous montrons que les résultats que nous avions obtenus s'étendent à tous les germes à lieu singulier de dimension 1 sans autre restriction. Ces invariants, essentiellement donnés par des (a,b)-modules géométriques, (objet qui est une abstraction du réseau de Brieskorn formel), décrivent les diverses connexions de Gauss-Manin filtrées associées à un tel germe, ainsi que les relations entre elles.

Texte reçu le 18 septembre 2008, accepté le 4 mai 2009

Daniel Barlet, Institut Élie Cartan UMR 7502, Nancy-Université, CNRS, INRIA et Institut Universitaire de France, BP 239 - F - 54506 Vandœuvre-lès-Nancy Cedex, France E-mail : barlet@iecn.u-nancy.fr

Classification mathématique par sujets (2000). - 32-S-25, 32-S-40, 32-S-50.

Mots clefs. - Hypersurfaces à singularités non isolées, germes holomorphes à lieu singulier de dimension 1, réseau de Brieskorn, $(a, b)$-module, connexion de Gauss-Manin filtrée. 
Abstract (On functions with a 1-dimensional singular locus). - In our previous paper [6] we constructed for a large class of germs of holomorphic functions $f:\left(\mathbb{C}^{n+1}, 0\right) \rightarrow(\mathbb{C}, 0)$ with one dimensional singular set $S:=\{d f=0\}$, analytic invariants which generalize the Brieskorn module of an isolated singularity germ. In the present article we show that all results obtained in this previous paper are valid for any holomorphic germ with one dimensional singular locus. So, these invariants, essentially given by geometric $(a, b)$-modules (this object is an "abstraction" of a formal Brieskorn module) describe the various filtered Gauss-Manin connections associated to such a germ, and the relations between them.

\section{Introduction}

1.1. Généralisation de l'étude locale aux points génériques de la courbe $S$. - Dans le présent article nous considèrerons la situation suivante :

Soit $f:\left(\mathbb{C}^{n+1}, 0\right) \rightarrow(\mathbb{C}, 0)$ un germe de fonction holomorphe dont le lieu singulier $S:=\left\{x / f(x)=0\right.$ et $\left.d f_{x}=0\right\}$ est un germe de courbe à l'origine, et soit $t:\left(\mathbb{C}^{n+1}, 0\right) \rightarrow(\mathbb{C}, 0)$ un germe de fonction holomorphe non singulière tel que la restriction de $t$ à $(S, 0)$ soit finie. Sur un voisinage ouvert $X$ de l'origine assez petit on aura pour chaque $\sigma \in S^{*}:=S \backslash\{0\}$ un (a,b)-module associé au germe en $\sigma$ de fonction à singularité isolée obtenu en restreignant $f$ à l'hypersurface lisse définie par $\{t=t(\sigma)\}$. Rappelons que ce $(\mathrm{a}, \mathrm{b})$-module n'est rien d'autre que le complété formel du module de Brieskorn de ce germe de fonction à singularité isolée (voir par exemple [7], [11], pour l'origine de cette notion et [3], [4] ou [5] pour les propriétés de base des (a,b)-modules).

Les faisceaux de cohomologie $\hat{\mathcal{H}} \bullet$ du complexe de de Rham

$$
\left(\hat{\mathrm{Ker}} d f^{\bullet}, d^{\bullet}\right)
$$

où Ker $d f^{\bullet}$ désigne le noyau de la multiplication extérieure par $d f$

$$
\wedge d f^{\bullet}: \hat{\Omega}^{\bullet} \rightarrow \hat{\Omega}^{\bullet+1}
$$

agissant sur le complété formel en $f$ des formes holomorphes, sont nuls en degrés différents de $1, n, n+1$ dans cette situation et les faisceaux $\hat{\mathcal{H}}^{n}$ et $\hat{\mathcal{H}}^{n+1}$ sont à support dans $S$. La considération du germe auxiliaire $t$ permet de calculer ces deux faisceaux comme respectivement noyau et conoyau d'une $t$-connexion compatible avec les opérations $a$ et $b$ de la façon suivante.

Soit (Ker $\left.d f / d^{\bullet},{ }^{\bullet}\right)$ le complexe de de Rham $t$-relatif complété formellement en $f$, et soit $\mathbb{E}:=\mathcal{H}^{n}\left(\hat{\mathrm{K} e r} d f \boldsymbol{\rho}^{\bullet}, d_{j}^{\bullet}\right)$. Ce faisceau est naturellement muni d'opérations $a$ et $b$ ainsi que d'une structure de $t^{-1}\left(\mathcal{O}_{D}\right)$-module, où $D$ est un disque de centre 0 assez petit dans $\mathbb{C}$. Nous définissons alors une $t^{-1}\left(\mathcal{O}_{D}\right)$-connexion

$$
b^{-1} . \nabla: \mathbb{P} \rightarrow \mathbb{E}
$$


où $\mathbb{P}:=\{x \in \mathbb{E} / \nabla(x) \in b . \mathbb{E}\}$, qui commute à $a$ et $b$.

La proposition suivante est démontrée dans [6, prop. 4.2.8].

Proposition 1.1.1. - On a une suite exacte naturelle de faisceaux de $\mathbb{C}_{X^{-}}$ modules à support $S$ :

$$
0 \rightarrow \hat{\mathcal{H}}^{n} \stackrel{u}{\rightarrow} \mathbb{P} \stackrel{b^{-1} \cdot \nabla}{\longrightarrow} \mathbb{E} \stackrel{v}{\rightarrow} \hat{\mathcal{H}}^{n+1} \rightarrow 0
$$

compatible aux opérations a et b sur ces faisceaux, où $u$ est induite par la projection évidente $\hat{\mathrm{K}}$ er $d f^{n} \rightarrow \hat{\Omega}_{/}^{n}$ et $v$ par la multiplication extérieure par $d t$.

L'objectif principal de cet article est de montrer le résultat «technique » suivant :

THÉORÈme 1.1.2. - Dans la situation précisée ci-dessus, on a

1. le faisceau $\hat{\mathcal{H}}^{n}$ est un système local de $(a, b)$-modules géométriques sur $S^{*}$;

2. la restriction à $S^{*}$ du faisceau $\hat{\mathcal{H}}^{n+1}$ vérifie la propriété de prolongement analytique suivante

(PA) Soient $V \subset U$ deux ouverts de $S^{*}$, avec $U$ connexe et $V$ non vide. Alors la restriction $\Gamma\left(U, \hat{\mathcal{H}}^{n+1}\right) \rightarrow \Gamma\left(V, \hat{\mathcal{H}}^{n+1}\right)$ est injective.

On expliquera comment ce résultat se déduit du théorème de finitude 4.1.1 après l'énoncé du théorème 4.1.1.

Précisons que la «pièce manquante » dans [6] pour traiter le cas général obtenu ici, c'est à dire pour montrer le théorème 1.1.2, est essentiellement contenue dans le paragraphe 2.3 .

Le point crucial est l'existence sur $S^{*}$ d'un $t^{-1}\left(\mathcal{O}_{D}[[b]]\right)$-réseau $\mathbb{G} \subset \mathbb{E}$ qui est stable par $b^{-1} \nabla$ et $a$.

Le paragraphe 3 explicite le lien avec les dérivations d'intégrales « à la Malgrange » (voir [8]) de formes holomorphes sur les cycles évanescents dans ce cadre à deux fonctions (à savoir $f$ et $t$ ). Ce paragraphe est destiné à éclairer le lecteur sur la signification de la connexion $\nabla$.

Le paragraphe 4 est consacré à la preuve du théorème de finitude, qui, après la proposition 2.3.2, est facile et utilise des résultats classiques sur les familles de fonctions à singularité isolée.

On termine en donnant un critère pour estimer le faisceau $\mathbb{G}$ qui est loin d'être facile à calculer et en explicitant des exemples simples.

En conclusion le théorème 1.1.2 montre que l'hypothèse $(\mathrm{HH})$ des théorèmes 4.3.1 et 4.3.2 de [6] ne sont pas utiles pour obtenir les propriétés souhaitées des faisceaux $\hat{\mathcal{H}}^{n}$ et $\hat{\mathcal{H}}^{n+1}$ pour un germe de fonction holomorphe à singularité de dimension 1.

Rappelons que cette hypothèse $(\mathrm{HH})$ implique que la singularité isolée transverse est localement constante le long de $S^{*}$, ce qui est assez restrictif, bien que 
cette situation soit assez commune, par exemple quand le germe à l'origine est quasi-homogène.

Le théorème 1.1.2 implique que l'ensemble des résultats de [6] est valable dans le cadre général d'un germe à lieu singulier de dimension 1. En particulier le théorème de finitude 5.2.1, le corollaire 5.2.3 ainsi que les théorèmes 6.2.1 et 6.4.1 sont démontrés sous cette seule hypothèse. Les énoncés généralisant les théorèmes 5.2.1 et 6.2.1 sont détaillés ci-après pour la commodité du lecteur.

\subsection{Quelques conséquences}

1.2.1. Rappels. - Dans ce qui suit $f$ désignera un germe à l'origine de $\mathbb{C}^{n+1}$ de fonction holomorphe qui est supposé réduit.

Pour $f$ réduite, on a un isomorphisme naturel compatible à $a$ et $b$,

$$
\left.E_{1} \otimes \mathbb{C}_{Y} \simeq \hat{\mathcal{O}}_{X} \cdot d f \cap \operatorname{Ker} d \simeq \mathcal{H}^{1}\left((\hat{\operatorname{Ker}} d f)^{\bullet}, d^{\bullet}\right)\right)
$$

où l'on a posé $E_{1}:=\mathbb{C}[[z]] . d z$, muni des opérations $a:=\times z$ et $b:=\left(\int_{0}^{z}\right) . d z$.

Placé en degré 1 ce faisceau défini un sous-complexe, que nous noterons $E_{1} \otimes \mathbb{C}_{Y}[1]$, du complexe $\left((\hat{\mathrm{K} e r} d f)^{\bullet}, d^{\bullet}\right)$.

Nous définirons le complexe $\left((\tilde{\mathrm{K} e r} d f)^{\bullet}, d^{\bullet}\right)$ comme le quotient

$$
\left((\tilde{\operatorname{Ker}} d f)^{\bullet}, d^{\bullet}\right):=\left((\hat{\operatorname{Ker}} d f)^{\bullet}, d^{\bullet}\right) / E_{1} \otimes \mathbb{C}_{Y}[1] .
$$

Notons $\tilde{\mathcal{A}}$ la $\mathbb{C}$-algèbre $\tilde{\mathcal{A}}:=\left\{\sum_{\nu=0}^{\infty} P_{\nu}(a) . b^{\nu}\right.$, avec $\left.P_{\nu} \in \mathbb{C}[x]\right\}$ dont le produit est défini par les deux conditions suivantes :

1) On a la relation de commutation $a \cdot b-b . a=b^{2}$.

2) La multiplication par $a$ (à gauche ou à droite) est continue pour la filtration $b$-adique.

On a alors le résultat général suivant (voir [6, th. 2.1.1]) :

ThÉORÈme 1.2.1. - Les complexes $\left((\tilde{\operatorname{Ker}} d f)^{\bullet}, d^{\bullet}\right)$ et $\left((\hat{\operatorname{Ker}} d f)^{\bullet}, d^{\bullet}\right)$ sont canoniquement quasi-isomorphes à des complexes de faisceaux de $\tilde{\mathcal{A}}$-modules (à gauche) sur $Y:=f^{-1}(0)$ ayant des différentielles $\tilde{\mathcal{A}}$-linéaires.

De plus, via ces quasi-isomorphismes, la suite exacte

$$
0 \rightarrow E_{1} \otimes \mathbb{C}_{Y}[1] \rightarrow\left((\hat{\operatorname{Ker}} d f)^{\bullet}, d^{\bullet}\right) \rightarrow\left((\tilde{\operatorname{Ker}} d f)^{\bullet}, d^{\bullet}\right) \rightarrow 0
$$

correspond à une suite exacte de complexes de faisceaux de $\tilde{\mathcal{A}}$-modules.

Ce théorème montre l'existence d'une structure naturelle de $\tilde{\mathcal{A}}$-modules sur tout groupe d'hypercohomologie de ces deux complexes. Il donne également la $\tilde{\mathcal{A}}$-linéarité (à gauche) des applications naturelles entre ces groupes. 
1.2.2. Les résultats principaux. — Supposons maintenant que le lieu singulier $S$ de $f$ est une courbe.

Rappelons qu'un $\tilde{\mathcal{A}}$-module $E$ est dit régulier géométrique s'il est de type fini sur la sous-algèbre $\mathbb{C}[[b]]$ de $\tilde{\mathcal{A}}$, et si son quotient par sa $b$-torsion (qui toujours est un $(\mathrm{a}, \mathrm{b})$-module, puisque $\mathbb{C}[[b]]$-libre de type fini) est un (a,b)module régulier géométrique.

Rappelons encore qu'un (a,b)-module $E$ est régulier si son saturé par $b^{-1} . a$, noté $E^{\sharp}$, est encore de type fini sur $\mathbb{C}[[b]]$. Il sera dit géométrique si, de plus, les valeurs propres de $b^{-1} . a$ agissant sur l'espace vectoriel de dimension finie $E^{\sharp} / b . E^{\sharp}$ sont dans $\mathbb{Q}^{+*}$ (donc rationnelles strictement positives). Cette condition est vérifiée pour le $(\mathrm{a}, \mathrm{b})$-module de Brieskorn d'un germe à singularité isolée grâce au théorème de monodromie et au théorème de positivité de Malgrange (voir [9]).

Notons $\hat{\mathcal{H}}^{i}$ le i-ème faisceau de cohomologie du complexe $\left((\hat{\operatorname{Ker}} d f)^{\bullet}, d^{\bullet}\right)$.

Nous noterons $c \cap S$ (resp. c) la famille des fermés de $Y$ qui rencontrent $S$ suivant un compact (resp. la famille des compacts de $Y$ ).

Le théorème 1.2.2 implique la généralisation suivante du théorème 5.2 .1 de [6].

ThÉORÈme 1.2.2. - Pour un germe $f:\left(\mathbb{C}^{n+1}, 0\right) \rightarrow(\mathbb{C}, 0)$ de fonction holomorphe à lieu singulier de dimension 1 les $\tilde{\mathcal{A}}$-modules suivants sont réguliers géométriques :

1. $H_{\{0\}}^{i}\left(Y, \hat{\mathcal{H}}^{j}\right)$ pour $i \geq 0$ et $j=1, n$.

2. $E:=H_{\{0\}}^{0}\left(Y, \hat{\mathcal{H}}^{n+1}\right)$.

3. $E_{\Phi}:=\mathbb{H}_{\Phi}^{n+1}\left(Y,\left((\hat{\operatorname{Ker}} d f)^{\bullet}, d^{\bullet}\right)\right)$ et $E_{\Phi}^{\prime}:=\mathbb{H}_{\Phi}^{n+1}\left(Y,\left((\tilde{\operatorname{Ker}} d f)^{\bullet}, d^{\bullet}\right)\right)$ pour $\Phi=c$ et $\Phi=c \cap S$.

On en déduit également la généralisation suivante du théorème 6.2.1 de [6].

ThÉORÈme 1.2.3. - On suppose $n \geq 2$. Pour un germe $f:\left(\mathbb{C}^{n+1}, 0\right) \rightarrow(\mathbb{C}, 0)$ de fonction holomorphe à lieu singulier de dimension 1 on a un accouplement (a,b)-sesquilinéaire naturel, non dégénéré

$$
h: E_{c \cap S}^{\prime} \times E \longrightarrow\left|\Xi^{\prime}\right|^{2}
$$

donné par intégration dans les fibres de $f$.

On a posé ici, pour $n \in \mathbb{N}$ fixé

$$
\left|\Xi^{\prime}\right|^{2}:=\sum_{r \in]-1,0] \cap \mathbb{Q}, j \in[0, n]} \mathbb{C}[[s, \bar{s}]] \cdot|s|^{2 r} \cdot\left(\log |s|^{2}\right)^{j} / \mathbb{C}[[s, \bar{s}]] .
$$

Précisons ce que signifie «donné par intégration sur les fibres de $f$ ». 
Soient $\omega$ et $\omega^{\prime} \operatorname{des}(n+1)$-formes $\mathcal{C}^{\infty}$ annulées par $\wedge d f$ et par $d$, le support de $\omega^{\prime}$ rencontrant $S$ suivant un compact $K$. Soit $\rho \in \mathcal{C}_{c}^{\infty}(X)$ vérifiant $\rho \equiv 1$ au voisinage de $K$. Alors nous définirons

$$
h\left(\left[\omega^{\prime}\right],[\omega]\right)=\frac{1}{(2 i \pi)^{n}} \int_{f=s} \rho \cdot \frac{\omega^{\prime}}{d f} \wedge \frac{\bar{\omega}}{d f} \in\left|\Xi^{\prime}\right|^{2} .
$$

Le calcul des $\tilde{\mathcal{A}}$-modules $E_{c \cap S}^{\prime}$ et $E$ via de telles formes $\mathcal{C}^{\infty}$ est justifié par le lemme 6.1.1 de [6].

Précisons enfin ce que nous entendons par accouplement «non dégénéré ».

- Pour tout élément $\left[\omega^{\prime}\right] \in E_{c \cap S}^{\prime}$ qui n'est pas de $b$-torsion, il existe un élément $[\omega] \in E$ tel que $h\left(\left[\omega^{\prime}\right],[\omega]\right) \neq 0$ dans $\left|\Xi^{\prime}\right|^{2}$.

- Pour tout élément $[\omega] \in E$ qui n'est pas de $b$-torsion, il existe un élément $[\omega] \in E_{c \cap S}^{\prime}$ tel que $h\left(\left[\omega^{\prime}\right],[\omega]\right) \neq 0$ dans $\left|\Xi^{\prime}\right|^{2}$.

\section{La $b^{-1} \cdot \nabla$ finitude}

2.1. La situation considérée. - Soit $f:\left(\mathbb{C}^{n+1}, 0\right) \rightarrow(\mathbb{C}, 0)$ un germe de fonction holomorphe à lieu singulier $(S, 0)$ de dimension 1 , et soit $t:\left(\mathbb{C}^{n+1}, 0\right) \rightarrow(\mathbb{C}, 0)$ un germe de fonction holomorphe non singulière. Notons $(\Sigma, 0)$ le lieu critique du germe d'application

$$
(f, t):\left(\mathbb{C}^{n+1}, 0\right) \rightarrow\left(\mathbb{C}^{2}, 0\right) .
$$

Faisons l'hypothèse que $(\Sigma, 0)$ soit de dimension 1 et que la restriction de $t$ à $(\Sigma, 0)$ soit finie.

Alors il existe un voisinage ouvert de Stein $X$ de l'origine dans $\mathbb{C}^{n+1}$ et un disque $D$ de centre 0 dans $\mathbb{C}$ vérifiant les propriétés suivantes :

1. Chaque composante irréductible de $S:=\left\{x \in X / f(x)=0\right.$ et $\left.d f_{x}=0\right\}$ contient l'origine, est non singulière en dehors de l'origine et est un disque topologique.

2. La restriction $t_{\mid \Sigma}: \Sigma \rightarrow D$ est un revêtement ramifié fini dont la restriction $t_{\mid \Sigma^{*}}: \Sigma^{*} \rightarrow D^{*}$ est un revêtement non ramifié, où nous avons posé $\Sigma^{*}:=\Sigma \backslash\{0\}$ et $D^{*}:=D \backslash\{0\}$.

L'existence de tels voisinages ouverts $X$ et $D$ de 0 dans $\mathbb{C}^{n+1}$ et $\mathbb{C}$ arbitrairement petits est élémentaire. L'existence, pour un germe $f$ donné, de germes holomorphes auxiliaires convenables est conséquence du lemme suivant :

LEMME 2.1.1. - Soit $f$ un germe de fonction holomorphe à l'origine de $\mathbb{C}^{n+1}$ dont le lieu singulier $S$ est de dimension 1. Pour $l \in\left(\mathbb{C}^{n+1}\right)^{*}$ dans un ouvert dense, le lieu critique $\Sigma_{l}$ du germe d'application $(f, l):\left(\mathbb{C}^{n+1}, 0\right) \rightarrow\left(\mathbb{C}^{2}, 0\right)$ est de dimension 1 et contient $S$; on a donc l'égalité $\Sigma_{l}=S$ au voisinage de $S^{*}$. 
De plus, on peut demander que la restriction de $l \grave{a} \Sigma_{l}$ soit propre et finie avec un unique point de ramification à l'origine.

Un tel $l$ étant fixé, il existe un disque ouvert $D$ centré à l'origine dans $\mathbb{C}$ assez petit, tel que la famille $\left(f_{\tau}\right)_{\tau \in D}$, où $f_{\tau}:=f_{\mid\{l=\tau\}}$, soit sur $D^{*}$ une famille $\grave{a} \mu$-constant le long de chaque composante connexe de $S^{*}$.

Démonstration. - Comme $(S, 0)$ est par hypothèse un germe de courbe, pour $l \in\left(\mathbb{C}^{n+1}\right)^{*}$ générique on aura $\{l=0\} \cap S=\{0\}$. Si, par exemple, $\left(\frac{\partial f}{\partial x_{1}}, \ldots, \frac{\partial f}{\partial x_{n}}\right)$ est une suite régulière en 0 , toute forme linéaire d'un ouvert dense dans un voisinage ouvert assez petit de la forme linéaire $x \mapsto x_{0}$ sera telle que le lieu critique de l'application $(f, l)$ sera de dimension 1 et n'aura pas de composante irréductible contenue dans $\{l=0\}$. On aura donc un ouvert dense sur lequel les deux premières conditions de l'énoncé sont réalisées avec, de plus, le fait que chaque fonction $f_{t}$ ne présente que des points singuliers isolés dans l'hyperplan $\{l=t\}$ pour tout $t$ assez voisin de 0 .

Le fait que $\mu$ reste localement constant sur $S^{*}$ au voisinage de l'origine est alors conséquence du fait que le faisceau $l_{*}\left(\Omega_{/ l}^{n} / d_{/ l} f \wedge \Omega_{/ l}^{n-1}\right)$ est cohérent sur $\mathcal{O}_{D}$ et donc localement libre en dehors de l'origine, puisque la restriction de $l$ au support de ce faisceau cohérent est finie.

2.2. Rappels. - Rappelons maintenant quelques constructions et quelques résultats donnés dans [6] pour l'étude de la «situation considérée ».

2.2.1. R.1. - Définissons le faisceau des formes $t$-relatives

$$
\Omega^{\bullet}:=\Omega_{X}^{\bullet} / d t \wedge \Omega_{X}^{\bullet-1}
$$

la différentielle $t$-relative étant induite par la différentielle de de Rham sur le quotient. Comme la fonction $t$ est non singulière on a un scindage naturel

$$
\Omega_{X}^{\bullet} \simeq d t \wedge \Omega_{X}^{\bullet-1} \oplus \Omega_{/}^{\bullet}
$$

ainsi qu'un isomorphisme différentiel gradué

$$
\wedge d t: \Omega^{\bullet} \rightarrow d t \wedge \Omega_{X}^{\bullet}
$$

Il est intéressant de remarquer que dans la suite exacte de cohomologie de la suite exacte courte de complexes

$$
0 \rightarrow\left(\Omega_{/}^{\bullet}, d_{j}^{\bullet}\right)[-1] \stackrel{\wedge d t}{\rightarrow}\left(\Omega_{X}^{\bullet}, d^{\bullet}\right) \rightarrow\left(\Omega_{/}^{\bullet}, d_{/}^{\bullet}\right) \rightarrow 0
$$

le connecteur s'identifie à la dérivation $\partial / \partial t$.

On a exactitude en degrés strictement positifs du complexe $\left(\Omega_{j}^{\bullet}, d_{j}^{\bullet}\right)$.

On a également exactitude en degrés $d \in[1, n-1]$ du complexe $\left(\Omega^{\bullet},(\wedge d / f)^{\bullet}\right)$ grâce à la finitude de $t: \Sigma \rightarrow D$.

Ces propriétés s'étendent immédiatement au complété formel $\hat{\Omega}^{\bullet}$ en $f$. 
2.2.2. R.2. - Introduisons maintenant le sous-complexe $\left((\hat{\operatorname{Ker}} d / f)^{\bullet}, d^{\bullet}\right) \mathrm{du}$ complexe $\left(\hat{\Omega}^{\bullet}, d^{\bullet}\right)$ où $\left(\hat{\mathrm{K} e r} d_{/} f\right)^{\bullet}$ est le noyau de la multiplication extérieure

$$
\wedge d / f: \hat{\Omega}_{j}^{\bullet} \rightarrow \hat{\Omega}_{/}^{\bullet+1} \text {. }
$$

Nous noterons respectivement par $\mathcal{E}$ et $\mathbb{E}$ les faisceaux de cohomologie de ce complexe en degrés 1 et $n$. Ils sont naturellement munis d'opérations $a$ et $b$ vérifiant $a . b-b . a=b^{2}$ déduites de la multiplication par $f$ et de $\wedge d_{/} f \circ\left(d_{/}\right)^{-1}$. Celles-ci commutent à l'action naturelle de $t^{-1}\left(\mathcal{O}_{D}\right)$ sur ces faisceaux.

On remarquera que la complétion formelle fait que ces faisceaux, à priori portés par $\Sigma$, sont à support dans $S$ puisque $S=\Sigma \cap\{f=0\}$, grâce à notre hypothèse sur $t$. En effet un germe de courbe irréductible $(\Gamma, 0)$ contenu dans $\Sigma \cap\{f=0\}$ vérifie ou bien $(\Gamma, 0) \subset(S, 0)$ ou bien $\Gamma \cap S=\{0\}$. Dans ce dernier cas, $\Gamma \backslash\{0\}$ est connexe et contenue dans l'ouvert lisse $\{d f \neq 0\}$ de $\{f=0\}$, et la fonction $t_{\mid \Gamma \backslash\{0\}}$ est localement constante, donc identiquement nulle. On en déduit que $(\Gamma, 0) \subset(\Sigma, 0) \cap\{t=0\}=\{0\}$, ce qui est absurde. D'où notre assertion.

Le théorème suivant a été établi dans [6].

THÉORÈme 2.2.1. - ([6, th. 4.2.1]) Dans la " situation considérée » au début de cette section, les faisceaux de cohomologie du complexe $\left(\left(\hat{\mathrm{Ker}} d_{/} f\right)^{\bullet}, d^{\bullet}\right)$ sont nuls en degrés différents de 1 et $n$. En degré 1 et $n$ les faisceaux $\mathcal{E}$ et $\mathbb{E}$ sont des $t^{-1}\left(\mathcal{O}_{D}[[b]]\right)$-modules cohérents, localement libres sur $S^{*}$, si le disque $D$ est assez petit.

2.2.3. R.3. - Dans la «situation considérée » définissons le morphisme de faisceaux de $\mathbb{C}$-espaces vectoriels sur $S$

$$
\nabla: \mathbb{E} \rightarrow \mathbb{E}
$$

en posant

$$
\nabla[d / \xi]:=\left[d / f \wedge \frac{\partial \xi}{\partial t}-\frac{\partial f}{\partial t} \cdot d \xi\right]
$$

La vérification du fait que ce morphisme de faisceaux est bien défini est facile. Les propriétés suivantes sont démontrées dans [6] lemme 4.2.5 et proposition 4.2 .8 .

Proposition 2.2.2. - Soit $\mathbb{P}:=\{x \in \mathbb{E} / \nabla(x) \in b . \mathbb{E}\}$. C'est un sous$t^{-1}\left(\mathcal{O}_{D}[[b]]\right)$-module de $\mathbb{E}$ qui est stable par a. Le morphisme de faisceaux

$$
b^{-1} \cdot \nabla: \mathbb{P} \rightarrow \mathbb{E}
$$

induit par $\nabla$ est une $t^{-1}\left(\mathcal{O}_{D}\right)$-connexion qui commute à a et $b$.

Son noyau et son conoyau sont respectivement canoniquement isomorphes comme $\tilde{\mathcal{A}}$-modules aux faisceaux de cohomologie $\hat{\mathcal{H}}^{n}$ et $\hat{\mathcal{H}}^{n+1}$ du complexe $\left(\hat{\mathrm{K}} \operatorname{er} d f^{\bullet}, d^{\bullet}\right)$. 
L'algèbre $\tilde{\mathcal{A}}$ qui n'intervient ici essentiellement que pour traduire la compatibilité aux opérations $a$ et $b$ de cet isomorphisme, a été définie (brièvement) au 1.2.1. Pour plus de détails sur cette algèbre nous renvoyons le lecteur à [4].

2.3. La $b^{-1} \cdot \nabla$-finitude. — Le contrôle du noyau de $\nabla$ sera obtenu grâce à la propriété suivante.

DéFInition 2.3.1. - On dira que $\mathbb{E}$ est $b^{-1} \nabla$-fini si, localement sur $D^{*}$, il existe un entier $N$ et un sous- $t^{-1}\left(\mathcal{O}_{D}[[b]]\right)$-module cohérent $\hat{G}$ de $\mathbb{P}$ qui est stable par $b^{-1} \nabla$ et contient $b^{N} . \mathbb{E}$.

Proposition 2.3.2. - Il existe sur $D$ un plus grand sous- $t^{-1}\left(\mathcal{O}_{D}[[b]]\right)-$ module $\mathbb{G}$ de $\mathbb{P}$, stable par $b^{-1} \nabla$.

Il vérifie de plus les propriétés suivantes:

(1) On $a \mathbb{G}=\left\{x \in \mathbb{E} / \forall \nu \in \mathbb{N}, \nabla^{\nu}(x) \in b^{\nu} . \mathbb{E}\right\}$.

(2) Si $x \in \mathbb{E}$ vérifie $b^{-1} \nabla(x) \in \mathbb{G}+t^{-1}\left(\mathcal{O}_{D}[[b]]\right) . x$ alors $x \in \mathbb{G}$.

En particulier, on a $\operatorname{Ker} \nabla \subset \mathbb{G}$.

(3) Si des germes $\varphi \in \mathcal{O}_{D} \backslash\{0\}$ et $x \in \mathbb{E}$ sont tels que $t^{-1}(\varphi) . x \in \mathbb{G}$ alors $x \in \mathbb{G}$.

(4) Si des germes $\varphi \in \mathcal{O}_{D} \backslash\{0\}$ et $x \in \mathbb{G}$ sont tels que $t^{-1}(\varphi) . x \in b$. $\mathbb{G}$ alors $x \in b$. $G$.

Supposons maintenant que $\mathbb{E}$ soit $b^{-1} \nabla$-fini sur $S^{*}$. Alors $\mathbb{G}$ est $t^{-1}\left(\mathcal{O}_{D}[[b]]\right)$ localement libre de rang fini sur $S^{*}$ et il est stable par a. De plus il vérifie les deux propriétés suivantes :

i) Le sous-faisceau $\operatorname{Ker} \nabla$ est localement constant sur $S^{*}$, de fibre un $(a, b)$ module régulier géométrique.

ii) Soit $U \subset D^{*}$ un ouvert connexe et simplement connexe, et soit $V \subset S^{*}$ une composante connexe de $t^{-1}(U)$. On a un isomorphisme

$$
\Gamma(V, \mathbb{G}) \simeq \Gamma(V, \operatorname{Ker} \nabla / b \cdot \operatorname{Ker} \nabla) \otimes_{\mathbb{C}} t^{-1}\left(\mathcal{O}_{D^{*}}(U)[[b]]\right) .
$$

2.3.1. Remarque. - Précisons que $\mathbb{G}$ est maximal au sens suivant : pour tout ouvert $\Omega \subset D$ et tout sous-faisceau $\Gamma$ de $\mathcal{O}_{D}[[b]]$-modules de $\mathbb{P}_{\mid \Omega}$ stable par $b^{-1} . \nabla$ on aura $\Gamma \subset \mathbb{G}$.

Démonstration. - Notons $\mathbb{G}_{k}:=\left\{x \in \mathbb{E} / \forall \nu \in[0, k] \nabla^{\nu}(x) \in b^{\nu} . \mathbb{E}\right\}$, pour $k \in \mathbb{N}$. On a donc $\mathbb{G}=\cap_{k \geq 0} \mathbb{G}_{k}$. Comme $\mathbb{G}_{k}$ est manifestement stable par $b$, pour voir que $\mathbb{G}_{k}$ est un sous- $\mathcal{O}_{D}[[b]]-$ module, il suffit de voir qu'il est stable par l'action de $t^{-1}\left(\mathcal{O}_{D}\right)$. 
Pour $\varphi \in \mathcal{O}_{D}$ et $x \in \mathbb{E}$ on a (en oubliant de noter l'image réciproque par $t$ )

$$
\nabla^{\nu}(\varphi \cdot x)=\sum_{j=0}^{\nu} C_{\nu}^{j} \cdot \varphi^{(j)} \cdot b^{j} \cdot \nabla^{\nu-j}(x)
$$

où $\varphi^{(j)}$ désigne la dérivée $j$-ème de $\varphi$.

Cette formule montre que si $x \in \mathbb{G}_{k}$ on aura $\varphi \cdot x \in \mathbb{G}_{k}$.

L'inclusion de tout sous- $t^{-1}\left(\mathcal{O}_{D}[[b]]\right)$-module stable par $b^{-1} \nabla$ dans $\mathbb{G}$ est immédiate. Pour prouver (1) il suffit donc de montrer que $\mathbb{G}$ est stable par $b^{-1} \nabla$. Or

$$
\nabla\left(\mathbb{G}_{k}\right)=\left\{\nabla(x) / x \in \mathbb{G}_{k}\right\} \subset\left\{y \in \mathbb{E} / \nabla^{\nu}(y) \in b^{\nu+1} \mathbb{E} \forall \nu \in[0, k-1]\right\}
$$

et ce dernier groupe est égal à

$$
\left\{b z / z \in \mathbb{E} / \nabla^{\nu}(z) \in b^{\nu} . \mathbb{E} \forall \nu \in[0, k-1]\right\}=b . \mathbb{G}_{k-1} .
$$

On a donc bien $\nabla(\mathbb{G}) \subset b$. $\mathbb{G}$.

$\mathrm{Si} \quad x \in \mathbb{E} \quad$ vérifie $\quad b^{-1} \nabla(x) \in \mathbb{G}+t^{-1}\left(\mathcal{O}_{D}[[b]]\right) \cdot x \quad$ considérons $G:=t^{-1}\left(\mathcal{O}_{D}[[b]]\right) \cdot x+\mathbb{G}$. On a alors, pour $y \in \mathbb{G}$,

$$
\nabla\left(t^{-1}(\alpha) \cdot x+y\right) \in t^{-1}\left(\alpha^{\prime}\right) \cdot b x+t^{-1}(\alpha) \cdot \nabla(x)+b \cdot \mathbb{G} \subset b \cdot\left(t^{-1}\left(\mathcal{O}_{D}[[b]]\right) \cdot x+\mathbb{G}\right)
$$

où $\alpha^{\prime}$ désigne la dérivée en $t \operatorname{de} \alpha \in \mathcal{O}_{D}[[b]]$. Ceci montre que $G=\mathbb{G}$ et donc que $x \in \mathbb{G}$. Ceci montre (2).

Si maintenant on a $t^{-1}(\varphi) \cdot x \in \mathbb{G}$ avec $\varphi \in \mathcal{O}_{D} \backslash\{0\}$ et $x \in \mathbb{E}$ montrons par récurrence sur $\nu \in \mathbb{N}$ que l'on a $\nabla^{\nu}(x) \in b^{\nu} . \mathbb{E}$. La propriété étant claire pour $\nu=0$ supposons-la vraie pour $\nu-1$ et montrons-la pour $\nu$. Comme on a la relation (@) on déduit de l'hypothèse $t^{-1}(\varphi) \cdot x \in \mathbb{G}$ et de l'hypothèse de récurrence que $t^{-1}(\varphi) \cdot \nabla^{\nu}(x) \in b^{\nu} . \mathbb{E}$. Mais sur $D^{*}$ le faisceau $\mathbb{E} / b^{\nu} . \mathbb{E}$ est localement $t^{-1}\left(\mathcal{O}_{D}\right)$-libre ${ }^{(1)}$ on en déduit que $\nabla^{\nu}(x) \in b^{\nu}$. $\mathbb{E}$. On en conclut que $x \in \mathbb{G}$, ce qui achève la preuve du point (3).

Le point (4) se montre de la même façon en utilisant l'égalité (déjà vue plus haut)

$$
b . \mathbb{G}=\left\{x \in \mathbb{E} / \forall \nu \in \mathbb{N}, \nabla^{\nu}(x) \in b^{\nu+1} . \mathbb{E}\right\} .
$$

Supposons maintenant que $\mathbb{E}$ est $b^{-1} \nabla$-fini.

Comme nos assertions sont locales sur $S^{*}$, nous pouvons nous placer audessus d'un disque $U \subset D^{*}$, considérer une composante connexe $V$ de $t^{-1}(U)$ et supposer que l'on ait sur $V$ un sous- $\mathcal{O}_{D}[[b]]$-module cohérent $\hat{G}$ contenant $b^{N} . \mathbb{E}_{\mid V}$ et stable par $b^{-1} \cdot \nabla$.

(1) Car c'est le cas pour $\nu=1$ et donc pour tout $\nu$ en raisonnant par récurrence sur la suite exacte

$$
0 \rightarrow b^{k} . \mathbb{E} / b^{k+1} . \mathbb{E} \rightarrow \mathbb{E} / b^{k+1} . \mathbb{E} \rightarrow \mathbb{E} / b^{k} . \mathbb{E} \rightarrow 0
$$

puisque $b^{k}: \mathbb{E} / b . \mathbb{E} \rightarrow b^{k} . \mathbb{E} / b^{k+1} . \mathbb{E}$ est un isomorphisme de faisceaux de $\mathcal{O}_{D}$-modules. 
Pour simplifier les notations, nous identifierons $U$ et $V$ via $t$.

2.3.2. Étape 1. - On se ramène au cas où le $\mathcal{O}_{D}$-module $\mathbb{P} / \hat{G}$ est libre sur $V$ et de rang noté $p$, et où, de plus, il est facteur direct du $\mathcal{O}_{D}$-module libre $\mathbb{E} / \hat{G}$ dont le rang sur $V$ sera noté $p+q$. Ceci est montré dans le lemme suivant.

LEMME 2.3.3. - On se place dans la situation précisée ci-dessus. Il existe un sous- $\mathcal{O}_{D}[[b]]$-module $\tilde{G}$ cohérent stable par $b^{-1} . \nabla$ qui contient $\hat{G}$ et qui vérifie de plus les propriétés suivantes:

a) Le $\mathcal{O}_{D}$-module $\mathbb{P} / \tilde{G}$ est libre.

b) La suite exacte de $\mathcal{O}_{D}$-modules

$$
0 \rightarrow \mathbb{P} / \tilde{G} \rightarrow \mathbb{E} / \tilde{G} \rightarrow \mathbb{E} / \mathbb{P} \rightarrow 0
$$

est scindée.

Démonstration. - Le quotient $\mathbb{P} / \hat{G}$ est $\mathcal{O}_{D}$-cohérent puisque $\hat{G}$ contient $b^{N} . \mathbb{P}$. Donc sa $\mathcal{O}_{D}$-torsion $T$ est cohérente sur $\mathcal{O}_{D}$. Si $\pi: \mathbb{P} \rightarrow \mathbb{P} / \hat{G}$ est l'application quotient, définissons

$$
\tilde{G}:=\pi^{-1}(T)
$$

Il est $\mathcal{O}_{D}[[b]]$-cohérent comme noyau et la propriété a) est évidente. Il contient $\hat{G}$ par définition. Montrons la stabilité par $b^{-1} . \nabla$.

Le problème est local au voisinage de chaque point $t_{0}$ appartenant au support du faisceau $T$, support qui est fermé et discret dans $V$. Près d'un tel point, on aura $x \in \tilde{G}_{t_{0}}$ si et seulement si il existe un entier $k \geq 1$ tel que l'on ait $\left(t-t_{0}\right)^{k} . x \in \hat{G}$. Alors l'égalité

$$
\left(t-t_{0}\right)^{k+1} \cdot b^{-1} \cdot \nabla(x)=b^{-1} \cdot \nabla\left(\left(t-t_{0}\right)^{k+1} \cdot x\right)-(k+1) \cdot\left(t-t_{0}\right)^{k} \cdot x
$$

montre que $b^{-1} \cdot \nabla(x) \in \tilde{G}$ puisque $\hat{G}$ est stable par $b^{-1} \cdot \nabla$.

Le scindage de la suite exacte est conséquence du fait que le $\mathcal{O}_{D}$-module $\mathbb{E} / \mathbb{P}$ est libre. En effet si on a $\varphi \cdot x \in \mathbb{P}$ avec $\varphi \neq 0$, cela signifie que l'on a $\nabla(\varphi \cdot x)=\varphi^{\prime} . b x+\varphi \cdot \nabla(x) \in b . \mathbb{E}, c^{\prime}$ est à dire que $\varphi \cdot \nabla(x) \in b . \mathbb{E}$. Comme $\mathbb{E} / b . \mathbb{E}$ est sans $\mathcal{O}_{D^{-}}$-torsion, cela signifie que $\nabla(x) \in b$. $\mathbb{E}$ c'est à dire que $x \in \mathbb{P}$.

On remplacera dans la suite $\hat{G}$ par $\tilde{G}$ mais en continuant à le noter $\hat{G}$.

Ayant choisi ces trivialisations, la connexion

$$
b^{-1} \cdot \nabla: \mathbb{P} / \hat{G} \rightarrow \mathbb{E} / \hat{G}
$$

se lit comme une $\mathcal{O}_{D}$-connexion associée à l'inclusion naturelle de $\mathcal{O}_{D}^{p}$ dans $\mathcal{O}_{D}^{p} \oplus \mathcal{O}_{D}^{q}$

$$
\partial: \mathcal{O}_{D}^{p} \rightarrow \mathcal{O}_{D}^{p} \oplus \mathcal{O}_{D}^{q}
$$

sur le disque $V$. 
2.3.3. Deuxième étape. — Elle est donnée par le lemme simple suivant.

LEMme 2.3.4. - Soit

$$
\partial: \mathcal{O}_{D}^{p} \rightarrow \mathcal{O}_{D}^{p} \oplus \mathcal{O}_{D}^{q}
$$

une $\mathcal{O}_{D}$-connexion sur un disque $V \subset D$. Alors Kerd est un sous-faisceau constant de $\mathbb{C}_{D}$-modules de $\mathcal{O}_{D}^{p}$ de rang au plus égal à $p$ et le plus grand sousfaisceau de $\mathcal{O}_{D}$-module stable par $\partial$ est égal à $\mathcal{O}_{D} . \mathrm{Ker} \partial$. Il est donc libre et facteur direct dans $\mathcal{O}_{D}^{p}$.

Démonstration. - Soit $\pi: \mathcal{O}_{D}^{p} \oplus \mathcal{O}_{D}^{q} \rightarrow \mathcal{O}_{D}^{p}$ la projection. Alors $\pi \circ \partial$ est une connexion sur $\mathcal{O}_{D}^{p}$ au-dessus du disque $V$. Soit $e:=\left(e_{1}, \ldots, e_{p}\right)$ une base horizontale pour cette connexion. Notons $\varepsilon:=\left(\varepsilon_{1}, \ldots, \varepsilon_{q}\right)$ la base canonique de $\mathcal{O}_{D}^{q}$. Définissons alors la matrice holomorphe $M: V \rightarrow L\left(\mathbb{C}^{p}, \mathbb{C}^{q}\right)$ par la formule

$$
\partial e=M \cdot \varepsilon \text {. }
$$

Pour $X \in \mathcal{O}_{D}^{p}$ on aura

$$
\partial\left({ }^{t} X . e\right)={ }^{t} X^{\prime} . e \oplus^{t} X . M . \varepsilon,
$$

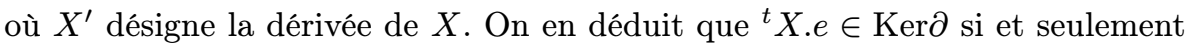
si $X \in \mathbb{C}^{p}$ et vérifie ${ }^{t} X . M \equiv 0$ sur $V$. Ceci définit un sous-espace vectoriel de $\mathbb{C}^{p}$ et montre donc la première assertion.

Les autres assertions sont immédiates.

2.3.4. Troisième étape. — Posons maintenant, avec les notations précédentes

$$
G:=\mathcal{O}_{D}[[b]] . K e r \partial+\hat{G} .
$$

Alors $G$ est $\mathcal{O}_{D}[[b]]$-cohérent comme somme de deux sous-faisceaux cohérents de $\mathbb{P}$ qui est cohérent (voir $[6,7.2]$ ). Comme $\mathbb{G} / \hat{G}$ est un sous- $\mathcal{O}_{D}$-module de $\mathbb{P} / \hat{G}$ qui est stable par $\partial$, on aura, d'après le lemme 2.3.4

$$
\mathbb{G} \subset G \text {. }
$$

Mais $G$ est un sous- $\mathcal{O}_{D}[[b]]$-module de $\mathbb{P}$ stable par $b^{-1} . \nabla$. En effet, si $x \in G$, comme on a

$$
b^{-1} \cdot \nabla(x) \in b^{-1} \cdot \nabla(\hat{G})+\mathcal{O}_{D} \cdot \operatorname{Ker} \partial+\hat{G} \subset G .
$$

On en conclut que $G=\mathbb{G}$ sur l'ouvert $V$. Ceci donne alors la cohérence de $\mathbb{G}$ sur $\mathcal{O}_{D}[[b]]$. Comme le faisceau $\mathbb{G} / b . \mathbb{G}$ est sans $\mathcal{O}_{D}$-torsion d'après la propriété (4) prouvée plus haut, on en conclut que $\mathbb{G}$ est (localement) libre de rang fini sur $\mathcal{O}_{D}[[b]]$. De plus, d'après la propriété (2) il contient $\operatorname{Ker} \nabla$.

Pour conclure, il suffit alors d'appliquer le théorème de Cauchy à la $\mathcal{O}_{D}[[b]]-$ connexion $b^{-1} . \nabla$ de $\mathbb{G}$. 


\section{Intégration «à la Malgrange »}

Ce chapitre est, bien sûr, directement inspiré de [8].

3.1. Complexe de de Rham absolu et $b^{-1} \cdot \nabla$. - Le lien entre le complexe de de Rham absolu $\left.(\hat{\mathrm{K} e r} d f)^{\bullet}, d^{\bullet}\right)$ et la connexion $b^{-1}$. $\nabla$ introduite plus haut est précisé par le lemme suivant

Lemme 3.1.1. - Soit $n$ un entier au moins égal à 2. On a un morphisme de complexes

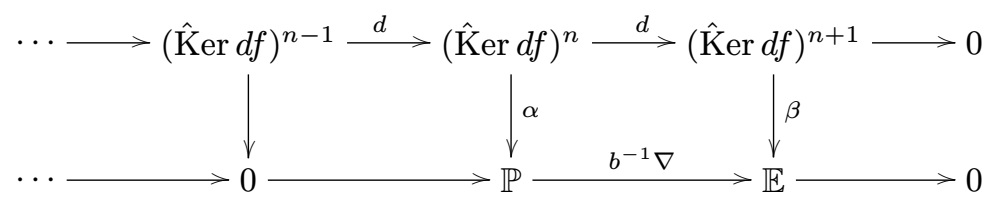

où $\alpha$ est induite par la projection «évidente » de $\hat{\Omega}^{n}$ sur $\hat{\Omega}_{/}^{n}$ et où $\beta$ est l'inverse de l'isomorphisme $\wedge d t: \hat{\Omega}^{n} \rightarrow \hat{\Omega}^{n+1}$. Il induit des isomorphismes (a,b)-linéaires sur les faisceaux de cohomologie, si on remplace $(\hat{\mathrm{K} e r} d f)^{0} \operatorname{par}(\hat{\mathrm{K} e r} d f)^{1} \cap \mathrm{Ker} d$ en degré 0 dans le premier complexe (avec l'inclusion évidente).

Démonstration. - Vérifions déjà les égalités $\alpha \circ d=0$ et $\beta \circ d=b^{-1} \nabla \circ \alpha$.

Si $u+d t \wedge v$ est dans $(\hat{\operatorname{Ker}} d f)^{n-1}$ avec $u \in \hat{\Omega}_{/}^{n-1}$ et $v \in \hat{\Omega}_{/}^{n-2}$, on aura $d_{/} f \wedge u=0$ et $(\alpha \circ d)(u+d t \wedge v)=d / u$. On trouve donc bien zéro dans $\mathbb{P} \subset \mathbb{E}$.

Pour $d / \xi+d t \wedge \eta \in(\hat{\operatorname{Ker}} d f)^{n}$ on aura

$$
(\beta \circ d)(d / \xi+d t \wedge \eta)=\beta\left(d t \wedge\left(\frac{\partial d / \xi}{\partial t}-d / \eta\right)\right)=\frac{\partial d / \xi}{\partial t}-d_{/} \eta=d_{/}\left(\frac{\partial \xi}{\partial t}-\eta\right) .
$$

La condition $d f \wedge(d / \xi+d t \wedge \eta)=0$ donne $\frac{\partial f}{\partial t} \cdot d / \xi=d_{/} f \wedge \eta$ et on a donc

$$
\nabla(\alpha(d / \xi+d t \wedge \eta))=\nabla(d / \xi)=d_{/} f \wedge\left(\frac{\partial \xi}{\partial t}-\eta\right)
$$

ce qui donne bien l'égalité désirée.

La fin de la preuve consiste alors à vérifier que les morphismes induits en cohomologie coïncident avec ceux de la proposition 1.1.1. Cette vérification simple est laissée au lecteur. 
3.2. Dérivations d'intégrales. - Comme près du point $p \in S^{*}$ la famille des fonctions à singularités isolées donnée par $t \rightarrow f_{t}$ est à $\mu$-constant elle est topologiquement triviale au voisinage de $p$; voir [13] et [14] pour le cas $n \neq 2$; pour $n=2$ on utilisera seulement la construction classique des fibrés en homologie ou en cohomologie pour laquelle l'invariance homotopique suffit (on pourra, par exemple, consulter [15] ou [16] à ce propos).

En identifiant $S^{*}$ et $D^{*}$ via la fonction $t$ au voisinage de $p$, on a donc l'existence d'un voisinage $U$ de $p$ dans $X$ et une application continue

$$
\Phi: U \rightarrow U_{p}:=t^{-1}(t(p)) \cap U
$$

donnant le diagramme commutatif

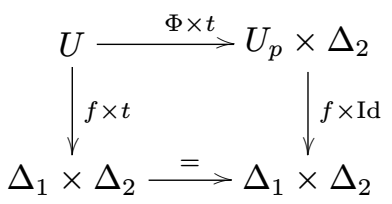

où $(\Phi \times t)$ est un homéomorphisme ${ }^{(2)}$ et $U_{p}$ une boule de Milnor en $p$ pour la restriction $f_{p}$ de $f$ à l'hyperplan $t^{-1}(t(p))$.

Soit $\gamma \in H_{n-1}\left(\{f=\varepsilon\} \cap U_{p}, \mathbb{C}\right)$, pour $\varepsilon>0$ assez petit. On a pour $0<|s|<\varepsilon$ et $t$ assez voisin de $t(p))$ une famille horizontale multiforme de $(n-1)$-cycles compacts contenus dans les fibres $\{f(t, x)=s\} \cap U$.

Étant donnée une section $\omega$ du faisceau $\mathbb{E}$ sur un voisinage ouvert de $p \in S^{*}$, nous définirons les intégrales «à la Malgrange »

$$
\int_{\gamma_{s, t}} \frac{\omega}{d / f}
$$

On remarquera déjà qu'une telle intégrale ne dépend que de la classe de $\omega$ dans $\mathbb{E}$ en raison de la formule de Stokes, puisque

$$
\mathbb{E}=\hat{\Omega}_{/}^{n} / d_{/}\left(\hat{\operatorname{Ker}} d_{/} f^{n-1}\right)=\hat{\Omega}_{/}^{n} / d_{/} f \wedge d / \hat{\Omega}_{/}^{n-2} .
$$

La proposition suivante exprime les dérivées partielles en $s$ et $t$ de ces fonctions holomorphes en $(s, t)$ qui sont multivaluées en $s$.

Proposition 3.2.1. - Pour tout $d_{/} \xi \in \mathbb{E}$ on a la formule de dérivation «en $s »$

que l'on peut lire sous la forme

$$
\frac{\partial}{\partial s}\left(\int_{\gamma_{s, t}} \xi\right)=\int_{\gamma_{s, t}} \frac{d / \xi}{d / f}
$$

$$
\text { primitive «en } s » d e\left(\int_{\gamma_{s, t}} \frac{d_{/} \xi}{d_{/} f}\right)=\int_{\gamma_{s, t}} \frac{d_{/} f \wedge \xi}{d_{/} f}=\int_{\gamma_{s, t}} \frac{b\left(d_{/} \xi\right)}{d_{/} f} .
$$

(2) Une équivalence d'homotopie suffit en fait. 
Pour tout $d / \xi \in \mathbb{E}$ on a la formule de dérivation « en $t$ »

$$
\frac{\partial}{\partial t}\left(\int_{\gamma_{s, t}} \xi\right)=\int_{\gamma_{s, t}} \frac{\nabla(d / \xi)}{d / f}
$$

que l'on peut lire sous la forme

$$
\frac{\partial}{\partial t}\left(\int_{\gamma_{s, t}} \frac{d / \xi}{d / f}\right)=\int_{\gamma_{s, t}} \frac{b^{-1} \nabla(d / \xi)}{d / f}
$$

pour $d / \xi \in \mathbb{P}$.

Démonstration. - Soit $\Delta \subset \mathbb{H} \times \mathbb{C}$ un polydisque. L'hypothèse de trivialité topologique permet de trouver $\psi \in \mathcal{C}_{c / f}^{\infty, n-1}\left(f^{-1}(\Delta)\right)$ une $(n-1)$-forme $d$-fermée induisant pour chaque $(s, t) \in \Delta$ la classe définie par $\gamma_{s, t}$ dans

$$
H_{c}^{n-1}\left(f_{t}^{-1}(s) \cap U, \mathbb{C}\right) \simeq H_{n-1}\left(f_{t}^{-1}(s) \cap U, \mathbb{C}\right) .
$$

Pour $\xi \in \Omega_{/}^{n-1}$ posons

$$
F(s, t):=\int_{\gamma_{s, t}} \xi=\int_{f_{t}^{-1}(s)} \xi \wedge \psi \quad \forall(s, t) \in \Delta .
$$

Commençons par prouver l'holomorphie de $F$ sur $\Delta$. Comme $F$ est manifestement une fonction continue, il suffit de prouver que son $\bar{\partial}$ au sens des distributions est nul. Considérons alors une forme test $\theta \in \mathcal{C}_{c}^{\infty,(2,1)}(\Delta)$. On a

$$
<\bar{\partial} F, \theta>=-<F, \bar{\partial} \theta>=-\int_{f^{-1}(\Delta)} \xi \wedge \psi \wedge d\left(f^{*}(\theta)\right)
$$

puisque $\bar{\partial} \theta=d \theta$ et puisque loin du lieu critique de $f$ le théorème de Fubini banal s'applique à des fonction continues. Comme on a

$$
\left.d\left(\xi \wedge \psi \wedge f^{*}(\theta)\right)=\xi \wedge \psi \wedge d\left(f^{*}(\theta)\right)\right)
$$

puisque $d \psi=0$ et que $d \xi \wedge f^{*}(\theta)$ est de type $(n+2,1)$ donc nulle, la formule de Stokes permet de conclure à l'holomorphie de $F$.

Pour calculer $\frac{\partial F}{\partial s}$ au sens des distributions, considérons maintenant une forme test $\zeta \in \mathcal{C}_{c}^{\infty,(0,2)}(\Delta)$. On aura

$$
<\frac{\partial F}{\partial s} \cdot d s, d t \wedge \zeta>=-<F, d(d t \wedge \zeta)>=-\int_{f^{-1}(\Delta)} \xi \wedge \psi \wedge d\left(f^{*}(d t \wedge \zeta)\right) .
$$

Comme on a $d \xi=d / \xi+d t \wedge \frac{\partial \xi}{\partial t}$ la formule de Stokes donne

$$
<\frac{\partial F}{\partial s} \cdot d s, d t \wedge \zeta>=\int_{f^{-1}(\Delta)} d / \xi \wedge \psi \wedge f^{*}(d t \wedge \zeta) .
$$

Le théorème de Fubini donne alors

$$
<\frac{\partial F}{\partial s} \cdot d s, d t \wedge \zeta>=\int_{\Delta}\left(\int_{f_{t}^{-1}(s)} \frac{d / \xi}{d / f} \wedge \psi\right) \cdot d s \wedge d t \wedge \zeta
$$


ce qui donne bien notre formule de dérivation «en $\mathrm{s}$ ».

Soit $\zeta$ une forme-test choisie comme plus haut. On a

$$
<\frac{\partial F}{\partial t} \cdot d t, d s \wedge \zeta>=-<F, d(d s \wedge \zeta)>=-\int_{f^{-1}(\Delta)} \xi \wedge \psi \wedge d\left(f^{*}(d s \wedge \zeta)\right) .
$$

La formule de Stokes donne alors

$<\frac{\partial F}{\partial t} \cdot d t, d s \wedge \zeta>=\int_{f^{-1}(\Delta)} \frac{\partial \xi}{\partial t} \wedge d t \wedge \psi \wedge d_{/} f \wedge f^{*}(\zeta)+d_{/} \xi \wedge \psi \wedge \frac{\partial f}{\partial t} . d t \wedge f^{*}(\zeta)$ puisque $f^{*}(d s)=d f=d_{/} f+\frac{\partial f}{\partial t}$. $d t$. On trouve alors, puisque

$$
\begin{gathered}
\nabla(d / \xi)=d_{/} f \wedge \frac{\partial \xi}{\partial t}-\frac{\partial f}{\partial t} \cdot d / \xi \\
<\frac{\partial F}{\partial t} . d t, d s \wedge \zeta>=\int_{f^{-1}(\Delta)} \nabla(d / \xi) \wedge \psi \wedge d t \wedge f^{*}(\zeta) .
\end{gathered}
$$

On a donc bien, à nouveau grâce au théorème de Fubini, la formule annoncée, pour $d_{/} \xi \in \mathbb{E}$ :

$$
\frac{\partial F}{\partial t}(s, t)=\int_{\gamma_{s, t}} \frac{\nabla(d / \xi)}{d / f} .
$$

Remarquons que si $I: \mathbb{E} \rightarrow \mathcal{O}_{\mathbb{H} \times \mathbb{C}}$ est le morphisme de faisceau localement défini par $[d / \xi] \rightarrow \int_{\gamma_{s, t}} \frac{d, \xi}{d / f}$, on a établi les égalités suivantes :

$$
\left(\partial_{s}\right)_{\circ} I_{\circ} b=I \text { et }\left(\partial_{t}\right)_{\circ} I=I_{\circ}\left(b^{-1} \nabla\right)
$$

de morphismes de faisceaux respectivement de $\mathbb{E}$ à valeurs dans $\mathcal{O}_{\mathbb{H} \times \mathbb{C}}$ et de $\mathbb{P}$ à valeurs dans $\mathcal{O}_{\mathbb{H} \times \mathbb{C}}$.

Reprenons les notations introduites au début de ce paragraphe. Le lemme suivant montre que les intégrales sur les cycles déterminent une section du faisceau $\mathbb{E}$.

Lemme 3.2.2. - Soit $p$ un point de $S^{*}$ et soit $\theta$ un germe en $p$ de section $d u$ faisceau $\mathbb{E}$ tel que pour tout couple $(s, t)$ assez voisin de $(0, t(p))$ dans $\mathbb{C}^{*} \times \mathbb{C}$ on ait

$$
\int_{\gamma_{s, t}^{j}} \frac{\theta}{d / f} \equiv 0
$$

pour une base horizontale $\gamma_{s, t}^{j}$ déduite d'une base de l'espace vectoriel

$$
H_{n-1}(\{f=\varepsilon\} \cap U, \mathbb{C}) \simeq H_{n-1}(\{f=\varepsilon\} \cap\{t=t(p)\} \cap U, \mathbb{C}) .
$$

Alors on a $\theta=0$ dans $\mathbb{E}$. 
Démonstration. - D'après notre hypothèse, pour chaque $t_{0}$ fixé assez voisin de $t(p)$ la section du fibré de Gauss-Manin de la fonction $f_{t_{0}}$ sur l'hyperplan $\left\{t=t_{0}\right\}$, induite par $\theta_{\mid\left\{t=t_{0}\right\}}$, est de torsion. Comme la fonction $f_{t_{0}}$ a une singularité isolée en $p\left(t_{0}\right):=S^{*} \cap t^{-1}\left(t_{0}\right)$, son fibré de Gauss-Manin est sans torsion d'après [12], et on a donc que la valeur de $\theta$ en $p\left(t_{0}\right)$ est nulle, c'est à dire que l'image de $\theta$ dans $\mathbb{E} /\left(t-t_{0}\right)$. $\mathbb{E}$ est nulle. Comme ceci a lieu pour tout $t_{0}$ assez voisin de $t(p)$ et comme le faisceau $\mathbb{E}$ est localement libre de rang fini sur $t^{-1}\left(\mathcal{O}_{D}[[b]]\right)$ sur $S^{*}$, on en conclut que $\theta$ est nulle au voisinage de $p$.

\section{Le théorème de $b^{-1} \cdot \nabla$-finitude sur $S^{*}$}

4.1. Preuve du théorème de finitude. - Le théorème 1.1.2 sera une conséquence simple du résultat suivant.

THÉORÈme 4.1.1. - Dans la «situation considérée » précisée au début du chapitre 2, $\mathbb{E}$ est $b^{-1}$. $\nabla$-fini sur $S^{*}$.

Preuve du théorème 1.1.2. - Il résulte de la proposition 2.3.2 que $\operatorname{Ker} \nabla$, qui d'après la proposition 1.1.1. est isomorphe à $\hat{\mathcal{H}}^{n}$ (rappelons que $\mathbb{G} \subset \mathbb{P}$ et que $\mathbb{E}$ est sans $b$-torsion), est localement constant sur $S^{*}$ et a pour fibre un (a,b)-module régulier géométrique.

De même, l'assertion ii) de la proposition 2.3.2. implique la propriété $(P A)$ pour le faisceau $\hat{\mathcal{H}}^{n+1}$ puisque ce faisceau est, d'après la proposition 1.1.1. isomorphe au conoyau de $b^{-1} \cdot \nabla$. On a donc une suite exacte

$$
0 \rightarrow \mathbb{P} / \mathbb{G} \stackrel{b^{-1} . \nabla}{\longrightarrow} \mathbb{E} / \mathbb{G} \rightarrow \hat{\mathcal{H}}^{n+1} \rightarrow 0
$$

où l'on sait que les faisceaux $\mathbb{E} / \mathbb{G}$ et $\mathbb{P} / \mathbb{G}$ sont des $\mathcal{O}_{D^{*}-\text { modules localement }}$ libres d'après le (3) de la proposition 2.3.2. et le lemme 2.3.3. Ceci permet de conclure.

Reprenons les notations du paragraphe 3.2.

Notons $q: \hat{\Omega}^{\bullet} \rightarrow \hat{\Omega}^{\bullet}$ l'application quotient et remarquons que l'on a l'égalité $d_{/} \circ q=q \circ d$. Nous noterons encore par $d_{/}: \hat{\Omega}^{\bullet} \rightarrow \hat{\Omega}_{/}^{\bullet+1}$ cette application composée. Donc pour $\omega \in \hat{\Omega}^{n-1}$ nous aurons l'élément $[d / \omega] \in \mathbb{E}$.

Si les formes $\omega, \omega^{\prime} \in \hat{\Omega}^{n-1}$ vérifient

$$
f . d \omega=d f \wedge \omega^{\prime}
$$

on aura dans $\mathbb{E}$ la relation $a .[d / \omega]=b \cdot\left[d / \omega^{\prime}\right]$. Ceci se vérifie facilement en utilisant l'identification de $\Omega^{\bullet}$ avec les formes ne présentant pas l'élément différentiel « $d t »$ et la décomposition

$$
\Omega^{\bullet}=\Omega_{j}^{\bullet} \oplus d t \wedge \Omega_{/}^{\bullet-1}
$$


Proposition 4.1.2. - Soit $p \in S^{*}$ et considérons une base de Jordan $e_{1}, \ldots, e_{k}$ d'un bloc de Jordan de la monodromie de $f$ pour la valeur propre $\exp (-2 i \pi . u)$ où $u \in\left[0,1\left[\right.\right.$, agissant sur $H^{n-1}(\{f=\varepsilon\} \cap U, \mathbb{C})$.

Alors il existe un entier $m \geq 0$ et des $(n-1)$-formes holomorphes $\omega_{1}, \ldots, \omega_{k}$ sur $U$ vérifiant les propriétés suivantes :

1. On a sur $U$ les relations $d \omega_{j}=(m+u) \frac{d f}{f} \wedge \omega_{j}+\frac{d f}{f} \wedge \omega_{j-1} \forall j \in[1, k]$ avec la convention $\omega_{0} \equiv 0$.

2. L'espace vectoriel engendré par les classes de cohomologie induites sur $\{f=\varepsilon\} \cap U$ par les $\omega_{j}$ est égal au sous-espace vectoriel engendré par $e_{1}, \ldots, e_{k}$ de $H^{n-1}(\{f=\varepsilon\} \cap U, \mathbb{C})$.

Dans ces conditions les sections de $\mathbb{E}$ induites par les formes $d_{/} \omega_{j}$ sont dans le sous-faisceau $\operatorname{Ker} \nabla_{\mid \Delta}$ et le sous- $\mathbb{C}[[b]]$-module qu'elles engendrent est libre de rang $k$ et stable par $a$. Sa fibre est le $(a, b)$-module à pôle simple de rang $k$ de $\mathbb{C}[[b]]$-base $\varepsilon_{1}, \ldots, \varepsilon_{k}$ avec $a . \varepsilon_{j}=(m+u) . b . \varepsilon_{j}+b . \varepsilon_{j-1}$ avec la convention $\varepsilon_{0}=0$.

Le sous-faisceau de $t^{-1}\left(\mathcal{O}_{\Delta}[[b]]\right)$-module engendré par $\left[d_{/} \omega_{1}\right], \ldots,\left[d_{/} \omega_{k}\right]$ est stable par a et libre de rang $k$ sur $t^{-1}\left(\mathcal{O}_{\Delta}[[b]]\right)$. Il est stable par $b^{-1} . \nabla$ et donc contenu dans $\mathbb{G}$.

Si on part d'une base de Jordan complète de l'espace vectoriel $H^{n-1}(\{f=\varepsilon\} \cap U, \mathbb{C})$, le sous-faisceau de $t^{-1}\left(\mathcal{O}_{\Delta}[[b]]\right)$-modules ainsi obtenu est encore libre de rang fini.

REMARQUE 4.1. - Grâce à notre trivialisation locale, pour chaque $p^{\prime} \in \Delta$, les restrictions de la base $e_{1}, \ldots, e_{k}$ à $\left\{f_{p^{\prime}}=\varepsilon\right\}$ induiront une base de Jordan d'un bloc de Jordan de taille $k$ pour la monodromie de $f_{p^{\prime}}$ agissant sur $H^{n-1}\left(\left\{f_{p^{\prime}}=\varepsilon\right\} \cap U, \mathbb{C}\right) \quad$ (resp. une base de Jordan complète de $H^{n-1}\left(\left\{f_{p^{\prime}}=\varepsilon\right\} \cap U, \mathbb{C}\right)$ si on part d'une base de Jordan complète de $\left.H^{n-1}(\{f=\varepsilon\} \cap U, \mathbb{C})\right)$.

Démonstration. - Commençons par remarquer que si $\omega \in \Omega^{n-1}$ on a

$$
d f \wedge d \omega=d t \wedge \nabla(d, \omega) .
$$

Puisque l'on a $d f \wedge d \omega_{j}=0, j \in[1, k]$ les classes $\left[d / \omega_{j}\right] \in \mathbb{E}$ sont donc bien dans $\operatorname{Ker} \nabla_{\mid \Delta}$.

L'existence de l'entier $m$ et des $(n-1)$-formes holomorphes vérifiant les propriétés (1.) et (2.) résulte de [1]. Les sections sur l'ouvert $\Delta$ du faisceau $\hat{\mathcal{H}}^{n}$ induites par les formes $d / \omega_{j}$ vérifient les relations :

$$
a .\left[d / \omega_{j}\right]=(m+u) \cdot b \cdot\left[d / \omega_{j}\right]+b \cdot\left[d_{/} \omega_{j-1}\right] \forall j \in[1, k]
$$

qui ne sont qu'une réécriture des relations (1.). 
Montrons l'indépendance sur $\mathcal{O}_{D}[[b]]$. Si on a, sur un ouvert connexe $\Delta^{\prime} \subset \Delta$, une relation

$$
\sum_{j=1}^{k} s_{j} \cdot\left[d_{/} \omega_{j}\right] \in d_{/}\left(\Gamma\left(\Delta^{\prime},\left(\hat{\operatorname{Ker}} d_{/} f\right)^{n-1}\right)\right.
$$

où $s_{j} \in \Gamma\left(\Delta^{\prime}, \mathcal{O}_{D}[[b]]\right)$, pour $j \in[1 . k]$, on obtient pour chaque $t \in \Delta^{\prime}$ une relation sur $\mathbb{C}[[b]]$ des classes correspondantes, ce qui donne la nullité des coefficients pour chaque $t \in \Delta^{\prime}$ fixé. En effet, pour $t \in \Delta^{\prime}$ fixé, ces classes sont indépendantes sur $\mathbb{C}\left\{f_{t}\right\}$ dans le système de Gauss-Manin la fonction $f_{t}$ grâce à la remarque qui suit l'énoncé de la proposition, et donc aussi dans le module de Brieskorn correspondant puisqu'il est sans torsion d'après [12]. On en déduit la même propriété dans son complété formel en $f_{t}$ par platitude de $\mathbb{C}\left[\left[f_{t}\right]\right]$ sur $\mathbb{C}\left\{f_{t}\right\}$, et celui-ci coïncide avec son complété formel en $b$, qui est le $(\mathrm{a}, \mathrm{b})$-module associé à $f_{t}$ égal à la fibre en $t$ de $\mathbb{E}$. L'indépendance sur $\mathbb{C}[[b]]$ en résulte.

L'inclusion dans $\mathbb{G}$ est immédiate puisque les $\left[d / \omega_{j}\right]$ sont dans $\operatorname{Ker} \nabla$ (voir prop. 2.3.2 (2)).

L'indépendance pour une base de Jordan complète s'obtient de façon analogue.

Démonstration du théorème 4.1.1. - Compte tenu de la proposition précédente, il nous suffit de montrer qu'il existe localement sur $S^{*}$ un entier $N$ tel que le $t^{-1}\left(\mathcal{O}_{\Delta}[[b]]\right)$-module $\tilde{\mathbb{G}} \subset \mathbb{G}$ construit à partir d'une base de Jordan de la monodromie de $f$ agissant sur l'espace vectoriel $H^{n-1}(\{f=\varepsilon\} \cap U, \mathbb{C})$ vérifie $b^{N} . \mathbb{E} \subset \tilde{\mathbb{G}}$. Ceci résulte du fait que si l'on considère la construction effectuée, on peut choisir, d'après [2], l'entier $m$ au plus égal à $n$. On obtient alors, pour chaque singularité isolée $f_{p^{\prime}}$, un réseau $\widetilde{\mathbb{G}}_{p^{\prime}}$ du réseau de Brieskorn $\mathbb{E}_{p^{\prime}}$ qui vérifie $b^{n} \cdot \mathbb{E}_{p^{\prime}} \subset \tilde{\mathbb{G}}_{p^{\prime}}$. Comme ceci a lieu pour chaque $p^{\prime}$ assez voisin de $p$, on en conclut que $b^{n} . \mathbb{E} \subset \tilde{\mathbb{G}} \subset \mathbb{G}$.

On notera que l'on pourrait aussi utiliser la $V$-filtration de MalgrangeKashiwara (voir [10]) et la symétrie du spectre d'une singularité isolée pour obtenir le résultat ci-dessus.

4.2. Estimation de $\mathbb{G}$. - La preuve du théorème de $b^{-1}$. $\nabla$-finitude montre que $\mathbb{G}$ contient $b^{n} . \mathbb{E}$ sur $S^{*}$. Il est intéressant de pouvoir améliorer cette «minoration » de $\mathbb{G}$ dans certains exemples. La proposition ci-dessous donne un tel critère.

Nous allons travailler près d'un point générique de $S^{*}$. Choisissons, près d'un tel point, un système de coordonnées locales $t, x_{1}, \ldots, x_{n}$ tel que l'on ait

$$
S^{*}=\left\{x_{1}=\cdots=x_{n}=0\right\} .
$$

L'idéal $\mathfrak{M}$ de $\mathcal{O}_{X}$ engendré par $x_{1}, \ldots, x_{n}$ est donc l'idéal réduit de $S^{*}$. 
Commençons par remarquer que l'on a, localement sur $S^{*}$, équivalence entre l'égalité $\mathbb{G}=\mathbb{E}$ et l'appartenance de $\frac{\partial f}{\partial t}$ à $J /(f)$, l'idéal jacobien relatif de $f$. En effet, dès que $\frac{\partial f}{\partial t} \notin J /(f)$, on a $d x \notin \mathbb{P}$ et donc, à fortiori $d x \notin \mathbb{G}$.

Proposition 4.2.1. - Notons $\mathcal{M}^{k}$, pour $k \geq 1$, l'image de $\mathfrak{M}^{k} . \hat{\Omega}_{/}^{n}$ dans $\mathbb{E}$.

Supposons qu'il existe un entier $k$ tel que

$$
\mathfrak{M}^{k} \cdot \frac{\partial f}{\partial t} \subset \mathfrak{M}^{k+1} . J_{/}(f)
$$

Alors le plus grand sous- $\mathcal{O}_{D}[[b]]$-module (cohérent) $\mathbb{G}$ de $\mathbb{E}$, stable par a et $b^{-1} \nabla$ contient $\mathcal{M}^{k}$.

Démonstration. - Nous allons montrer que sous notre hypothèse $G:=\mathcal{M}^{k}$ est stable par $a$ et $b$ et il vérifie $\nabla(G) \subset b . G$.

La stabilité par $a$ est évidente. Pour montrer la stabilité par $b$, nous allons montrer que $b . G$ est l'image dans $\mathbb{E}$ de $\mathfrak{M}^{k+1} . J /(f) . \Omega_{/}^{n}$, ce qui implique en particulier la stabilité par $b$.

Si $\omega=d / \xi$ avec $\omega \in \mathfrak{M}^{k} . \Omega_{/}^{n}$, on peut choisir $\xi \in \mathfrak{M}^{k+1} . \Omega_{/}^{n-1}$. On aura alors $b[\omega]=\left[d_{/} f \wedge \xi\right]$ qui est bien dans $\mathfrak{M}^{k+1} . J_{/}(f) . \Omega_{/}^{n}$.

Réciproquement, si $\eta \in \mathfrak{M}^{k+1} . J_{/}(f) . \Omega_{/}^{n}$ on peut écrire $\eta=d_{/} f \wedge \xi$ avec $\xi \in \mathfrak{M}^{k+1} . \Omega_{/}^{n-1}$. Alors $d / \xi \in \mathfrak{M}^{k} . \Omega_{/}^{n}$ et donne un élément $[d / \xi] \in G$ dont l'image par $b$ est $[\eta]$. Ceci prouve notre assertion.

On a alors, pour $\omega=d / \xi$,

$$
\nabla(\omega)=d_{/ f} \wedge \frac{\partial \xi}{\partial t}-\frac{\partial f}{\partial t} \cdot \omega .
$$

Comme pour $[\omega] \in G$ on peut choisir $\xi$ et donc $\frac{\partial \xi}{\partial t} \in \mathfrak{M}^{k+1} \cdot \Omega_{/}^{n-1}$, on obtient, grâce à l'inclusion $\left.{ }^{*}\right), \nabla(\omega) \in \mathfrak{M}^{k+1} . J /(f) . \Omega_{/}^{n} \subset b . G$.

\subsection{Exemples}

4.3.1. Exemple 1. - On se propose d'étudier le cas où $f(t, x)=P(x)+t . Q(x)$ avec $P$ et $Q$ deux germes de fonctions holomorphes à l'origine de $\mathbb{C}^{n}$.

Lemme 4.3.1. - Supposons $P$ à singularité isolée à l'origine et supposons que l'on ait $\mathfrak{M}^{k+1} . J(Q) \subset \mathfrak{M}^{k+1} . J(P)$ pour un entier $k \geq 0$, où $\mathfrak{M}$ désigne l'idéal maximal de l'origine dans $\mathbb{C}^{n}$.

Alors on a $\widehat{\mathfrak{M}}^{k+1} . J /(f)=\widehat{\mathfrak{M}}^{k+1} . J(P)$ où $\widehat{\mathfrak{M}}$ désigne l'idéal engendré par $\mathfrak{M}$ dans $\mathcal{O}_{\mathbb{C}^{n+1}}$.

Si on a de plus $Q \in \mathfrak{M} . J(Q)$, ce qui est vérifié en particulier si $Q$ est quasi homogène, on obtiendra l'inclusion $\widehat{\mathfrak{M}}^{k} \cdot \frac{\partial f}{\partial t} \subset{\widehat{\mathfrak{M}^{k}}}^{k+1} . J /(f)$ et la proposition précédente donnera que $\mathcal{M}^{k} \subset \mathbb{G}$ sur $S=\{x=0\}$ au voisinage de l'origine. 
Démonstration. - L'hypothèse permet donc d'écrire chaque $x^{\alpha} \cdot \frac{\partial Q}{\partial x_{j}}$ pour $\alpha \in \mathbb{N}^{n}$, vérifiant $|\alpha|=k+1$, comme combinaison linéaire à coefficients holomorphes des $x^{\beta} \cdot \frac{\partial P}{\partial x_{i}}$. Si $\gamma$ désigne le vecteur colonne des $x^{\alpha} \cdot \frac{\partial P}{\partial x_{j}}$ et $\delta$ le vecteur colonne $\operatorname{des} x^{\alpha} \cdot \frac{\partial Q}{\partial x_{j}}$ on aura donc $\delta=\mathcal{R} \cdot \gamma$ où $\mathcal{R}$ est une matrice à coefficients holomorphes dans $\mathbb{C}^{n}$.

Comme les $x^{\alpha} \cdot\left[\frac{\partial P}{\partial x_{j}}+t \cdot \frac{\partial Q}{\partial x_{j}}\right]$ forment un système générateur sur $\mathcal{O}_{\mathbb{C}^{n+1}}$ de $\widehat{\mathfrak{M}}^{k+1} . J_{/}(f)$, on aura une relation matricielle

$$
\Gamma=(\operatorname{Id}+t \cdot \mathcal{R}) \cdot \gamma
$$

où $\Gamma$ désigne le vecteur colonne des $x^{\alpha} \cdot\left[\frac{\partial P}{\partial x_{j}}+t \cdot \frac{\partial Q}{\partial x_{j}}\right]$. Pour $|t| \ll 1$ la matrice Id $+t . \mathcal{R}$ sera inversible et on a ainsi établi l'égalité $\widehat{\mathfrak{M}}^{k+1} . J /(f)=\widehat{\mathfrak{M}}^{k+1} . J(P)$ au voisinage de l'origine dans $\mathbb{C}^{n+1}$. Cette égalité montre que le lieu singulier de $f$ est contenu dans $S=\{x=0\}$ au voisinage de l'origine, et comme on a $Q(0)=0$, égalité imposée par l'appartenance de $Q$ à $\mathfrak{M} . J(Q)$, on aura égalité. On conclut immédiatement car $Q \in J /(f)$ implique $Q \in J(P)$.

Illustrons ceci par un exemple simple « explicite».

4.3.2. Exemple 2. - Il s'agit de calculer l'exemple suivant dans lequel on a $n=2$ :

$P(x, y)=x^{4}+y^{4}, Q(x, y)=x^{2} \cdot y^{2}$ et donc $f(x, y, t)=P(x, y)+t . Q(x, y)$ qui est une déformation à $\mu$-constant pour $t \neq \pm 2$, d'hypersurfaces à singularités isolées de $\mathbb{C}^{2}$ dont le faisceau des modules de Brieskorn n'est pas localement constant. En effet le birapport des quatre droites de $\mathbb{C}^{2}$ que l'on obtient pour chaque valeur de $t$ et qui vaut ${ }^{(3)}-\frac{t-2}{4}$ est une fonction localement injective, ce qui montrent que ces germes de fonctions holomorphes ne sont jamais localement deux à deux analytiquement équivalentes.

Cependant l'hypothèse $\mathfrak{M}^{2} . J(Q) \subset \mathfrak{M}^{2} . J(P)$ du lemme précédent est satisfaite, où ici, on a simplement $\mathfrak{M}:=(x, y)$.

On vérifie aussi immédiatement que $Q \notin J(P)$. On aura donc $\mathbb{G}=\mathcal{M}$.

Explicitons le calcul de $\nabla$.

On a

$$
\begin{aligned}
& \frac{\partial f}{\partial x}=4 x^{3}+2 t . x y^{2} \\
& \frac{\partial f}{\partial y}=4 y^{3}+2 t . x^{2} y .
\end{aligned}
$$

(3) Il s'agit de calculer le birapport des quatre racines de l'équation $z^{4}+t . z^{2}+1=0$. Ce nombre est défini modulo le groupe du birapport. Par exemple $1-\left(-\frac{t-2}{4}\right)=\frac{t+2}{4}$ représente la même classe de birapport. 
et donc

$$
\begin{aligned}
8 . x^{3} y & =2 y \frac{\partial f}{\partial x}-t x \cdot\left(\frac{\partial f}{\partial y}-2 t . x^{2} y\right) \\
& =2 t^{2} \cdot x^{3} y+2 y \frac{\partial f}{\partial x}-t x \cdot \frac{\partial f}{\partial y} .
\end{aligned}
$$

On obtient ainsi

$$
\begin{aligned}
& 2\left(4-t^{2}\right) \cdot x^{3} y=2 y \frac{\partial f}{\partial x}-t x \cdot \frac{\partial f}{\partial y} \in J_{/}(f) \text { et } \\
& 2\left(4-t^{2}\right) \cdot x y^{3}=2 x \frac{\partial f}{\partial y}-t y \cdot \frac{\partial f}{\partial x} \in J_{/}(f) \text { par symétrie. }
\end{aligned}
$$

On en déduit que

$$
\begin{aligned}
& 4 . x^{5}=x^{2} \cdot \frac{\partial f}{\partial x}-2 t . x^{3} y^{2} \in \in J_{/}(f) \text { et } \\
& 4 . y^{5}=y^{2} \cdot \frac{\partial f}{\partial y}-2 t . x^{2} y^{3} \in J_{/}(f) \text { par symétrie. }
\end{aligned}
$$

Grâce aux relations

$$
\begin{aligned}
& 4 . x^{4}=-2 t \cdot x^{2} y^{2}+x \cdot \frac{\partial f}{\partial x} \\
& 4 . y^{4}=-2 t . x^{2} y^{2}+y \cdot \frac{\partial f}{\partial y}
\end{aligned}
$$

une $\mathcal{O}_{D}[[b]]$-base de $\mathbb{E}$ est donc donnée par

$$
\left(1, x, y, x^{2}, y^{2}, x y, x^{2} y, x y^{2}, x^{2} y^{2}\right) . d x \wedge d y .
$$

Soit $\omega:=x . d y-y . d x$. Pour un monôme $m$ homogène de degré $\delta(m)$ on a

$$
d_{/}(m . \omega)=\frac{\delta(m)+2}{4} \cdot \frac{d_{/} f}{f} \wedge m . \omega
$$

ce qui donne

$$
a(m)=\frac{\delta(m)+2}{4} \cdot b(m) .
$$

On a également

$$
\begin{array}{r}
\nabla(d(m . \omega))=d / f \wedge \frac{\partial(m \cdot \omega)}{\partial t}-x^{2} y^{2} \cdot d(m . \omega) \\
\text { et donc } \nabla(m)=-x^{2} y^{2} \cdot m .
\end{array}
$$

Comme $x^{2} y^{2} \cdot \mathfrak{M} \subset J_{/}(f)$, on voit que $b^{-1} \nabla$ opère sur $\mathbb{G}=\mathcal{M}$.

Par exemple, comme

$$
\begin{aligned}
2\left(4-t^{2}\right) \cdot x^{3} y^{2} & =2 y^{2} \frac{\partial f}{\partial x}-t x y \cdot \frac{\partial f}{\partial y} \\
& =d_{/} f \wedge\left(2 y^{2} d y+t . x y d x\right)
\end{aligned}
$$

TOME $137-2009-\mathrm{N}^{\circ} 4$ 
on aura

$$
b^{-1} \nabla(x)=\frac{t . x}{2\left(4-t^{2}\right)} .
$$

Donc $\left(4-t^{2}\right)^{\frac{1}{4}} \cdot x$ sera (localement) dans Ker $\nabla$ pour $|t|<2$.

Comme $\nabla(1)=-x^{2} y^{2} \notin b$. $\mathbb{E}$, pour tester directement la $b^{-1} \nabla$-finitude de $\mathbb{E}$, posons $E_{1}:=\mathbb{E} \oplus \mathcal{O}_{D} \cdot \varepsilon$, où l'on définit $\varepsilon:=b^{-1}\left(x^{2} y^{2}\right)$.

Alors on obtient, puisque $a\left(x^{2} y^{2}\right)=\frac{3}{2} b\left(x^{2} y^{2}\right)$ les relations suivantes :

$$
a \varepsilon=\frac{1}{2} . b \varepsilon \text { et } \nabla \varepsilon=b^{-1} \nabla\left(x^{2} y^{2}\right)=b^{-1}\left(-x^{4} y^{4}\right) .
$$

Explicitons $b^{-1}\left(-x^{4} y^{4}\right)$. On a d'après (1)

$$
2\left(4-t^{2}\right) \cdot x^{4} y^{4}=d_{/} f \wedge\left(2 x y^{4} d y+t \cdot x^{2} y^{3} d x\right)
$$

ce qui donne $2\left(4-t^{2}\right) \cdot x^{4} y^{4}=b\left(2 y^{4}-3 t \cdot x^{2} y^{2}\right)$ et donc d'après (6)

$$
2\left(4-t^{2}\right) \cdot x^{4} y^{4}=b\left(-4 t \cdot x^{2} y^{2}+d / f \wedge\left(-\frac{1}{2} y \cdot d x\right)\right)=b\left(-4 t \cdot x^{2} y^{2}+\frac{1}{2} \cdot b(1)\right) .
$$

On a donc

$$
b^{-1} \nabla(\varepsilon)=\frac{2 t}{4-t^{2}} \cdot \varepsilon-\frac{1}{4\left(4-t^{2}\right)} \cdot 1 .
$$

Ceci permet de conclure que $E_{1}$ est stable par $b^{-1} \nabla$.

On obtient ainsi directement la $b^{-1} \nabla$-régularité pour $\mathbb{E}$ dans cet exemple.

Le $\mathcal{O}_{D}[[b]]$-module $\mathcal{O}_{D}[[b]] .1 \oplus \mathcal{O}_{D}[[b]] . \varepsilon$ est stable par $a$ et $b^{-1} \nabla$, via les formules :

$$
\begin{aligned}
a . \varepsilon & =\frac{1}{2} . b \varepsilon, a \cdot 1=\frac{1}{2} \cdot b .1 \\
b^{-1} \nabla(1) & =-\varepsilon, b^{-1} \nabla(\varepsilon)=\frac{2 t}{4-t^{2}} \cdot \varepsilon-\frac{1}{4\left(4-t^{2}\right)} .1 .
\end{aligned}
$$

4.3.3. Exemple 3. - Soient $p, q, r$ trois entiers $\geq 3$ tels que $\frac{1}{p}+\frac{1}{q}+\frac{1}{r}<1$ et posons

$$
f(x, y, z, t)=x^{p}+y^{q}+z^{r}+t x y z .
$$

Remarquons que l'on a $f \in \mathfrak{M}^{3}$ et donc $J_{/}(f) \subset \mathfrak{M}^{2}$.

Montrons que, sur l'ouvert $S^{*}:=\{t \neq 0\}$ de $\mathbb{C}$, les hypothèses de la proposition 4.2.1 sont vérifiées avec $k=1$. D'abord on a

$$
J_{/}(f)=\left(p . x^{p-1}+t . y z, q \cdot y^{q-1}+t . x z, r . z^{r-1}+t . x y\right) .
$$

Les relations

$$
\frac{\partial f}{\partial x}=p \cdot x^{p-1}+t . y z \frac{\partial f}{\partial y}=q \cdot y^{q-1}+t . x z \frac{\partial f}{\partial z}=r . z^{r-1}+t . x y
$$


donnent

$$
\begin{aligned}
p q . x^{p-1} \cdot y^{q-1} & =t^{2} \cdot x y z^{2}+\mathfrak{M}^{2} . J /(f) \text { ainsi que } \\
t . x^{p-1} \cdot y^{q-1} & =-r . z^{r-1} \cdot x^{p-2} y^{q-2}+\mathfrak{M}^{2} . J_{/}(f)
\end{aligned}
$$

puisque $x^{p-2} y^{q-2} \in \mathfrak{M}^{2}$. On a donc

$$
t^{3} . x y z^{2}+p q r . z^{r-1} \cdot x^{p-2} y^{q-2} \in \mathfrak{M}^{2} . J /(f)
$$

ou encore, puisque $p, q, r$, sont au moins égaux à 3 et $t \neq 0$,

$$
t^{3} . x y z^{2}\left(1+\frac{p q r}{t^{3}} . z^{r-3} x^{p-3} y^{q-3}\right) \in \mathfrak{M}^{2} . J /(f) .
$$

On aura aussi

$$
\begin{aligned}
t . x^{p} y & =-r . x^{p-1} z^{r-1}+x^{p-1} \cdot \frac{\partial f}{\partial z} \\
t^{2} \cdot x^{p} y & =-r . t . x^{p-1} z^{r-1}+\mathfrak{M}^{2} . J /(f)=r q \cdot y^{q-1} \cdot x^{p-2} \cdot z^{r-2}+\mathfrak{M}^{2} . J_{/}(f) \\
& =r q . y^{2} \cdot x \cdot z \cdot\left(y^{q-3} \cdot x^{p-3} \cdot z^{r-3}\right)+\mathfrak{M}^{2} . J /(f) \in \mathfrak{M}^{2} . J_{/}(f)
\end{aligned}
$$

d'après (@). On a donc, en utilisant encore le fait que $x, y, z$ jouent le même rôle, que

$$
\mathfrak{M} . \frac{\partial f}{\partial t} \subset \mathfrak{M}^{2} . J /(f) \text { ainsique } \mathfrak{M} . f \subset \mathfrak{M}^{2} . J /(f) .
$$

Il nous reste seulement à voir que $\frac{\partial f}{\partial t} \cdot h \in J_{/}(f)$ implique $h \in \mathfrak{M}$; ceci résulte du fait que $\frac{\partial f}{\partial t} \notin J /(f)$.

LEMME 4.3.2. - Notons par $G:=\mathfrak{M} . \Omega_{/}^{n} / d / f \wedge d / \Omega_{/}^{n-2}$.

Comme le fibré vectoriel sur $S^{*}$ défini par $\mathbb{E} / b . \mathbb{E}$ est trivial, on constate facilement que le $\mathcal{O}_{S}[[b]]$-module

$$
\Gamma:=G+\mathcal{O}_{S}[[b]] . b^{-1} \alpha
$$

de $\mathbb{E}\left[b^{-1}\right]$ où $\alpha:=x y z$, est stable par $a, b, b^{-1} \nabla$ et $b^{-1} a$ sur $S^{*}$.

Démonstration. - Comme on a

$$
p . x^{p}=q . y^{q}=r . z^{r}=-t . \alpha \operatorname{modulo} \mathfrak{M} . J_{/}(f),
$$

on aura

$$
f-(1-\rho) . t \alpha \in \mathfrak{M} . J_{/}(f) \text { avec } \rho=\frac{1}{p}+\frac{1}{q}+\frac{1}{r} .
$$

On en déduit en particulier que $a . \alpha \in \mathfrak{M}^{2} . J_{/}(f)$ et donc que $a b^{-1} \alpha$ est bien dans $\Gamma$. La stabilité par $b^{-1} a$ en découle alors puisque la relation $\mathfrak{M} . f \subset$ $\mathfrak{M}^{2} . J /(f)$ donne

$$
a . G \subset \mathfrak{M}^{2} . J /(f) \subset b . G \text {. }
$$




\subsubsection{Remarques}

1. Le cas $p=q=r=3$ relève du premier exemple traité plus haut.

2. Les cas $\frac{1}{p}+\frac{1}{q}+\frac{1}{r}<1$ résulte de l'étude de [6] puisque l'on a

$$
W:=\frac{1}{p} \cdot x \cdot \frac{\partial}{\partial x}+\frac{1}{q} \cdot y \cdot \frac{\partial}{\partial y}+\frac{1}{r} \cdot z \cdot \frac{\partial}{\partial z}+(1-\rho) \cdot t \cdot \frac{\partial}{\partial t}
$$

qui vérifie $W . f=f$ et ne s'annule pas $\operatorname{sur}\{t \neq 0\}$.

3. La relation $\mathfrak{M} . f \subset \mathfrak{M}^{2} . J /(f)$ implique que le plus grand sous-(a,b)module à pôle simple de $f_{t}$ pour chaque $t \neq 0$ est la fibre en $t$ de $\mathcal{M}$.

On a donc $\mathbb{G}=\mathcal{M}=\mathfrak{M}$. $\mathbb{E}$ dans ce cas. Donc $\mathcal{M}_{t=1}$ est le plus grand sous-(a,b)-module à pole simple de $\mathbb{E}_{t=1}$, d'après la description de $\mathcal{H}^{n}$ dans le cas où il existe un champ de vecteur holomorphe ne s'annulant pas et vérifiant $W . f=f$ près des points de $S^{*}$ (voir [6, théorème 4.3.1]).

\section{BIBLIOGRAPHIE}

[1] D. BARlet - «Contribution effective de la monodromie aux développements asymptotiques », Ann. Sci. École Norm. Sup. 17 (1984), p. 293-315.

[2] _ « Monodromie et pôles du prolongement méromorphe de $\int_{X}|f|^{2 \lambda} \square »$, Bull. Soc. Math. France 114 (1986), p. 247-269.

[3] _ « Theory of $(a, b)$-modules. I», in Complex analysis and geometry, Univ. Ser. Math., Plenum, 1993, p. 1-43.

[4] _ « Théorie des $(a, b)$-modules. II. Extensions », in Complex analysis and geometry (Trento, 1995), Pitman Res. Notes Math. Ser., vol. 366, Longman, 1997, p. 19-59.

[5] _ _ «odules de Brieskorn et formes hermitiennes pour une singularité isolée d'hypersurface », in Singularités, Inst. Élie Cartan, vol. 18, Univ. Nancy, 2005, p. 19-46.

[6] _ _ « Sur certaines singularités non isolées d'hypersurfaces II », à paraître au J. of Alg. Geom.

[7] E. BRIESKORN - «Die Monodromie der isolierten Singularitäten von Hyperflächen », Manuscripta Math. 2 (1970), p. 103-161.

[8] B. Malgrange - «Intégrales asymptotiques et monodromie », Ann. Sci. École Norm. Sup. 7 (1974), p. 405-430.

[9] _ _ «e polynôme de Bernstein d'une singularité isolée », in Fourier integral operators and partial differential equations (Colloq. Internat., Univ. Nice, Nice, 1974), Lecture Notes in Math., vol. 459, Springer, 1975, p. $98-119$. 
[10] _ « Polynômes de Bernstein-Sato et cohomologie évanescente », in Analysis and topology on singular spaces, II, III (Luminy, 1981), Astérisque, vol. 101-102, Soc. Math. France, 1983, p. 243-267.

[11] M. Saito - «On the structure of Brieskorn lattice », Ann. Inst. Fourier (Grenoble) 39 (1989), p. 27-72.

[12] M. Sebastiani - «Preuve d'une conjecture de Brieskorn », Manuscripta Math. 2 (1970), p. 301-308.

[13] J. G. Timourian - « The invariance of Milnor's number implies topological triviality », Amer. J. Math. 99 (1977), p. 437-446.

[14] L. D. Tráng \& C. P. RAmanujam - «The invariance of Milnor's number implies the invariance of the topological type », Amer. J. Math. 98 (1976), p. $67-78$.

[15] A. N. VARChENKO - « Gauss-Manin connection of isolated singular point and Bernstein polynomial », Bull. Sci. Math. 104 (1980), p. 205-223.

[16] _ « The complex singularity index does not change along the stratum $\mu=$ const », Funktsional. Anal. i Prilozhen. 16 (1982), p. 1-12, 96. 